\title{
La littératie en santé, une compétence en mal de traitement
}

\section{Health literacy, a competency that is a matter of concern}

\section{Claude RICHARD ${ }^{1}$ et Marie-Thérèse LUSSIER ${ }^{1,2}$}

1 Équipe de recherche en soins de première ligne, Cité de la santé de Laval, Centre de santé et de services sociaux de Laval 1755, Bd René-Laennec, H7M 3L9 Laval, Québec, Canada

2 Département de médecine familiale, Faculté de médecine, Université de Montréal

Manuscrit reçu le 26 août 2008; commentaires éditoriaux formulés aux auteurs le 24 décembre 2008; accepté pour publication le 15 avril 2009

\section{Mots clés : Littératie; santé; médecins ; formation}

Keywords: Literacy; health; doctors; training
Résumé - Contexte : Selon les résultats d'enquêtes récentes sur la littératie chez les adultes, plus de la moitié des Canadiens adultes et près de $90 \%$ des personnes âgées ne possède pas un niveau de littératie assez élevé pour bien comprendre la posologie de médicaments ni les consignes de sécurité qui leur sont données. Par ailleurs, la situation se détériore avec l'âge mais aussi avec l'état de santé : plus on est malade et moins le niveau de littératie en santé est élevé. Les résultats suggèrent également que des problèmes de littératie en santé se retrouvent à tous les niveaux d'éducation. Exégèse : Or, le niveau de littératie en santé est le meilleur prédicteur de l'état de santé d'une personne. Il a été observé que les difficultés de lecture semblent avoir une influence sur l'observance, le respect des traitements, l'hospitalisation, la compréhension de la maladie et la compréhension du consentement aux soins. Conclusion : Les professionnels de la santé sont conscients depuis peu de l'importance de ces enjeux. Après avoir développé une expertise médicale, il est maintenant suggéré que le médecin en développe une nouvelle, non pour s'adresser à ses pairs mais pour s'adresser à son patient qui, une fois sur deux, ne possède pas le niveau de littératie en matière de santé nécessaire pour comprendre.

Abstract - Background: According to the results of recent surveys on adult literacy, more than $50 \%$ of adult and about $90 \%$ of elderly Canadians do not have the literacy level required to understand treatment posologies or security instructions given to them. Also, it seems the literacy level declines with age and the health status: the sicker people are, the least literate they are. In addition, results indicate that low health literacy levels are found in patients of different education levels, even among patients who pursued university diplomas. Exegesis: Health literacy level is considered to be the best predictor of an individual's health status. In fact, it has been observed that reading difficulties have an influence on adherence and respect of treatments, hospitalisation, understanding of the illness and understanding of treatment consent. Conclusion: Health professionals are becoming more and more aware of the importance of these issues. Authors are suggesting that doctors complement their medical expertise by a training to develop skills to communicate in a comprehensive way with their patients, who may not have the health literacy level required to fully understand them. 


\section{Introduction}

La littératie est un concept multidimensionnel qui va bien au-delà de la simple capacité de lire et d'écrire et qui, en ce sens, étend considérablement le concept d'alphabétisme. Il s'agit de "la capacité de comprendre et d'utiliser la lecture, l'écriture, la parole et d'autres moyens de communication pour participer à la société, atteindre ses objectifs personnels et donner sa pleine mesure » ${ }^{[1]}$. On met aujourd' hui en exergue la nécessité pour une personne de disposer d'une telle capacité pour gérer de façon optimale les problèmes de santé auxquels elle peut être confrontée et on insiste sur les difficultés que peuvent rencontrer des patients déficitaires pour l'une ou l'autre des compétences reliées à la littératie.

\section{Le concept de littératie : des compétences de quatre types}

Comme le rappelle Gauthier ${ }^{[2]}$, la littératie peut se diviser en quatre grands groupes de compétences, exposés dans le tableau I : 1) la compréhension des textes suivis (compétences pour comprendre et utiliser l'information de textes suivis); 2) la compréhension des textes schématiques (compétences pour le repérage et l'utilisation d'information présentée sous diverses formes) ; 3) la numératie (compétences permettant de composer avec les exigences mathématiques de diverses situations) et 4) la résolution de problèmes (compétences permettant de comprendre le problème et de le résoudre de manière planifiée et raisonnée).

Ces groupes de compétences ont été déterminés et évalués dans le cadre de plusieurs grandes enquêtes portant sur la littératie dans les pays industrialisés ${ }^{[3-5]}$, qui ont conduit à proposer de mesurer les compétences concernées à l'aide d'une échelle. Ainsi, les résultats de ces enquêtes sont présentés sous la forme d'un score global ou de scores individuels pour chacun des quatre domaines de compétence. Les scores varient de 0 à 500 et sont transposés en cinq niveaux de compétence, représentant des tâches de difficultés grandissantes ${ }^{[5]}$, résumés dans le tableau II. Au premier niveau, une personne a de grande difficulté à lire. Au second niveau, elle est capable de lire l'information de base et d'exécuter des tâches simples. Au troisième niveau, elle peut utiliser du matériel écrit de façon claire et exécuter des tâches élémentaires. C'est le niveau minimal requis pour répondre aux exigences de la vie en société. Enfin, au quatrième et au cinquième niveaux, la personne peut remplir la plupart des exigences de lecture et traiter de l'information complexe et difficile.

\section{La littératie en santé : des patients potentiellement en difficulté}

De façon plus détaillée, les enquêtes révèlent que le niveau 3 de compétence n'est pas atteint chez $42 \%$ de la population pour la compétence compréhension de textes suivis et schématiques, chez $55 \%$ pour la compétence en numératie et chez près de $70 \%$ pour la solution de problèmes. Or, lire les informations sur une notice explicative d'un médicament nécessite des capacités non négligeables comme celle de lire un tableau à double entrée, par exemple; la compréhension des posologies demande également de pouvoir prendre en compte l'âge et le poids et d'être capable de distinguer le type de médicament (gouttes, comprimés, etc.). De plus, lorsque les caractéristiques du patient ne correspondent pas aux spécifications du texte, il doit être capable d'ajuster le dosage par lui-même, ce qui n'est pas toujours évident, même pour un universitaire... Ainsi se pose la question de la littératie en matière de santé, dans un contexte où il est de première importance de rendre le patient plus autonome et responsable de sa santé.

Conscient des difficultés de lecture et de compréhension rencontrées par les patients, le Conseil canadien de l'apprentissage (CCA) a développé la «Health Activity Literacy Scale (HALS) », dans le but de permettre une mesure spécifique de la littératie en matière de santé, qui correspond à « la capacité d'une personne d'obtenir, de traiter et de comprendre l'information de base sur la santé et sur les 
Tableau I. Quatre groupes de compétences reliées au concept de littératie.

\begin{tabular}{|l|l|}
\hline 1- Compréhension de textes suivis & $\begin{array}{l}\text { Connaissances et compétences nécessaires pour comprendre et } \\
\text { utiliser l'information contenue dans des textes suivis, tels des } \\
\text { éditoriaux, des nouvelles, des brochures et des modes d'emploi. }\end{array}$ \\
\hline 2- Compréhension de textes schématiques & $\begin{array}{l}\text { Connaissances et compétences requises pour repérer et utiliser } \\
\text { l'information présentée sous diverses formes, par exemple les } \\
\text { consignes pour la prise de médicaments, les formulaires, les } \\
\text { fiches de paie, les horaires de transport, les cartes, les tableaux } \\
\text { et les graphiques. }\end{array}$ \\
\hline 3- Numératie & $\begin{array}{l}\text { Connaissances et compétences requises pour composer avec les } \\
\text { exigences mathématiques de diverses situations. Par exemple : } \\
\text { évaluation de ses risques d'avoir des effets secondaires. }\end{array}$ \\
\hline 4- Résolution de problèmes & $\begin{array}{l}\text { Réflexions ou actions orientées vers un but dans une situation } \\
\text { pour laquelle il n'existe pas de solution explicite. Lorsque l'ob- } \\
\text { jectif est défini, mais la façon de l'atteindre n'est pas évidente de } \\
\text { prime abord. Il s'agit donc de tâches qui impliquent : 1) la com- } \\
\text { préhension du problème et 2) sa résolution graduelle au moyen } \\
\text { d'une planification et d'un raisonnement. }\end{array}$ \\
\hline
\end{tabular}

Tableau II. Les niveaux de compétence dans la population générale, appréciés selon les scores globaux de littératie (appréciés de 0 à 500).

\begin{tabular}{|l|l|l|l|}
\hline Niveau & Score global & $\%$ population & Définition \\
\hline 1 & $0-225$ & $22 \%$ & $\begin{array}{l}\text { Grande difficulté ou incapacité à lire } \\
\text { sonne peut être incapable de déterminer correctement la dose de } \\
\text { médicaments à administrer d'après le mode d'emploi indiqué sur } \\
\text { l'emballage. }\end{array}$ \\
\hline 2 & $226-275$ & $26 \%$ & $\begin{array}{l}\text { Capacité à lire des écrits simples, explicites, et d'accomplir des } \\
\text { tâches simples comme repérer un mot familier }{ }^{[6]}\end{array}$ \\
\hline 3 & $276-325$ & $33 \%$ & $\begin{array}{l}\text { Capacité à utiliser du matériel écrit à condition qu'il soit clair } \\
\text { et à exécuter des tâches simples }{ }^{[6]} \text {. Il s'agit du niveau minimal } \\
\text { requis pour composer avec les exigences de la vie quotidienne } \\
\text { et du travail dans une société complexe. Il dénote à peu près } \\
\text { le niveau de compétences nécessaires pour obtenir un diplôme } \\
\text { d'études secondaires et accéder aux études supérieures. }\end{array}$ \\
\hline $4 / 5$ & $326-375$ & $20 \%$ & $\begin{array}{l}\text { Capacité à répondre à la plupart des exigences de lecture avec } \\
\text { beaucoup de polyvalence } \\
\text { ter de l'information complexe et exigeante. }\end{array}$ \\
\hline
\end{tabular}

services dont elle a besoin pour prendre des décisions éclairées sur sa santé » ${ }^{[3,6,8]}$.

Les données des enquêtes qui portent spécifiquement sur la littératie en santé indiquent des résultats encore plus médiocres que pour la littératie mesurée globalement ${ }^{[3-7,9]}$. Ainsi, en matière de littératie en santé, $55 \%$ (vs. $48 \%$ pour les enquêtes générales) de la population n'atteignent pas le niveau 3. Qui plus est, la situation se détériore avec l'âge mais aussi avec l'état de santé : plus on est malade et moins le niveau de littératie en santé est élevé. C'est donc dire que les personnes qui ont le plus besoin des outils intellectuels nécessaires pour se donner et obtenir des soins adéquats sont celles qui sont les plus démunies à cet égard.

La notion qu'il existe un lien entre le niveau de littératie et la santé est maintenant démontrée ${ }^{[10]}$. En fait, un faible taux en littératie est considéré comme 
une barrière dans le soin au patient ${ }^{[11]}$. Les patients avec un faible niveau de littératie comprennent moins bien les informations sur la santé, font moins de prévention et utilisent plus souvent les services d'urgence ${ }^{[8]}$. La plupart du matériel écrit produit dans le domaine de la santé pour un auditoire grand public nécessite en fait un niveau de scolarisation équivalent à des études du niveau de la fin de cycle secondaire canadien pour être bien compris; or, les enquêtes indiquent un niveau moyen de littératie dans la population qui correspond à des études du niveau de la fin du cycle primaire, voire inférieur. Qui plus est, plus de la moitié (55\%) des Canadiens ne possède pas le niveau de littératie en matière de santé nécessaire pour bien comprendre la posologie de médicaments, ni les consignes de sécurité qui leur sont données ${ }^{[1,12]}$. Cette proportion frise même les $90 \%$ chez les personnes âgées.

Par ailleurs, il est démontré que les patients avec un bas niveau de littératie n'approchent pas la communication de la même façon que ceux ayant un haut niveau de littératie. Un faible niveau de littératie est associé à une plus grande difficulté à assimiler de nouvelles informations et de nouveaux concepts et à une plus grande difficulté à communiquer avec le médecin ${ }^{[13,14]}$. Dans le cas du cancer, un bas niveau de littératie est associé à moins d'examens, des diagnostics tardifs, une diminution de l'acceptation du traitement et de l'observance et une diminution de la participation à des recherches cliniques. Des résultats similaires ont été trouvés chez les patients atteints de diabète ${ }^{[15]}$. En résumé, un faible taux de littératie compromet grandement la prise en charge de la maladie chronique par le patient ${ }^{[16]}$, notamment pour les maladies comme l'hypertension, le diabète et l'asthme ${ }^{[17]}$. Au contraire, un niveau élevé en littératie favorise un bon contrôle de maladie, comme par exemple le diabète ${ }^{[18]}$.

On remarque également que les personnes ayant des difficultés de lecture sont moins susceptibles de faire des examens préventifs, qu'elles sont hospitalisées plus souvent, qu'elles ont moins de connaissances concernant le traitement de leur maladie chronique et, dans les faits, qu'elles suivent moins bien les traitements prescrits ${ }^{[1,19]}$. Williams et al. ${ }^{[20]}$ ont trouvé que $42 \%$ de la population qu'ils ont étudiée n'étaient pas capables de comprendre les instructions relatives à la synchronisation de la prise du médicament et des repas, telles que celles concernant la nécessité de prendre un médicament «sur un estomac vide », c'est-à-dire à jeun ou, au contraire, en présence d'aliments, et que $26 \%$ n'arrivaient pas à comprendre la feuille de prise de rendez-vous. Ainsi, un individu n'ayant pas un niveau de littératie suffisant en santé n'est pas seulement limité dans sa capacité à comprendre les traitements, mais également dans sa capacité à aller chercher les soins nécessaires ou à s'orienter dans la bureaucratie médicale. Cette difficulté s'étend à la capacité de calculer (risques, probabilités, chances de ...), ce qui représente une barrière à la compréhension car la médecine se pratique à partir d'un contexte scientifique, de probabilités calculées sur une population.

\section{Implications en matière de formation des professionnels de la santé}

La prise de conscience qu'une difficulté en terme de littératie pouvait avoir des conséquences importantes sur la santé est relativement récente ${ }^{[1]}$. En effet, dans le milieu médical, on concevait qu'il suffisait de s'exprimer clairement et avec exactitude pour que le patient comprenne. On s'attendait également à ce que le patient se débrouille pour comprendre et on postulait que c'était sa responsabilité. On croyait qu'il y avait concordance entre le niveau d'éducation et celui de la littératie en santé. Les enquêtes ont montré que ce n'est pas tout à fait le cas ${ }^{[8]}$ et on identifie des problèmes de littératie en santé à tous les niveaux d'éducation.

Les professionnels de la santé sont conscients depuis peu de cette difficulté et de son impact sur l'observance, le respect des traitements ${ }^{[21]}$, la compréhension de la maladie et la compréhension du consentement aux soins ${ }^{[19]}$. Auparavant, ces faits revêtaient peu d'importance car la médecine s'exerçait dans un contexte autoritaire, ou tout au moins 
paternaliste, où il était attendu que le patient se conforme aux recommandations du médecin. Après avoir développé une expertise médicale tant au niveau de la pratique que du discours, il faut maintenant que le médecin en développe une nouvelle, non pour s'adresser à ses pairs, mais pour s'adresser à ses patients ${ }^{[22,23]}$.

Or, la formation professionnelle dans le domaine de la santé vise en très grande partie à faire maîtriser le langage et le vocabulaire de la spécialité médicale. Cette maîtrise d'un vocabulaire désignant des phénomènes physiopathologiques ou des parties de l'anatomie humaine (rarement considérées par un non professionnel de la santé) est, en bonne partie, ce qui caractérise le spécialiste en santé. Cette maîtrise lui sert à communiquer avec ses pairs et à échanger des informations avec les différents professionnels concernés mais cette formation spécialisée peut devenir un obstacle lorsque le spécialiste doit communiquer avec un non-spécialiste ${ }^{[23]}$. La complexité du langage médical peut influencer la perception de la gravité de la maladie. Ainsi, un patient peut se croire atteint d'une maladie plus rare et plus grave que celle dont il souffre en réalité, avec toutes les conséquences négatives que cela implique ${ }^{[24]}$. Le fait de développer des stratégies communicationnelles adaptées aux différents niveaux de littératie des patients dans la formation des médecins serait susceptible d'améliorer l'état de santé des patients et de promouvoir une approche plus collaborative de la médecine ${ }^{[23]}$.

Lever ces obstacles doit être une priorité, quand on sait que le niveau de littératie en santé est le meilleur prédicteur de l'état de santé et de mortalité d'une personne, meilleur que le revenu, l'éducation, la profession, etc. ${ }^{[25]}$ Il est donc particulièrement important d'ajouter à la formation des spécialistes en santé un volet qui leur permettra de mieux communiquer avec les non-spécialistes, qu'il s'agisse des patients ou de leurs aidants naturels. On peut ajouter que cet effort de communication en direction du patient est sans doute aujourd'hui plus nécessaire qu'autrefois où savoir lire, écrire et compter de manière rudimentaire était considéré comme suffisant.
Actuellement, il est évident que cela n'est plus le cas : des maladies chroniques comme le diabète nécessitent un niveau de littératie élevé pour que le patient puisse y faire face efficacement ${ }^{[16]}$. Les patients doivent être capables de lire et comprendre une ordonnance, les fiches descriptives de produits, les mises en garde et avoir un vocabulaire de base pour se représenter correctement le monde médical. Le dernier cours de biologie suivi (et parfois le seul) remonte au collège pour la plupart des personnes et ce cours portait sur la biologie générale et non sur les différentes pathologies humaines et leur traitement. La maîtrise des problèmes bio-cliniques que le professionnel de santé acquiert lors de sa formation doit donc désormais être complétée par celle des problèmes de communication avec les patients qui, une fois sur deux, ne possèdent pas le niveau de littératie en matière de santé nécessaire pour comprendre ${ }^{[1]}$. En effet, il faut que les professionnels puissent expliquer en termes clairs et compréhensibles des renseignements complexes. En d'autres mots, ils doivent être capables de vulgariser le jargon médical.

Enfin, les enquêtes conduites en matière de littératie en santé démontrent, si besoin était, que la communication avec un patient n'est pas « un long fleuve tranquille ${ }^{[19]}$. On mesure alors mieux les difficultés de communication que peuvent rencontrer des médecins face, par exemple, à des migrants ou des expatriés, quand entrent en ligne de compte des différences de maîtrise de la langue, des variantes culturelles mais aussi des écarts de parcours éducatifs et des degrés de maîtrise différents des modes de formalisation de l'information. C'est dire l'importance pour le médecin de fournir des soins de santé culturellement appropriés ${ }^{[26]}$.

\section{Hypothèses de solutions et pistes pour la mise en œuvre opérationnelle}

La littératie en santé rejoint donc les préoccupations pratiques de l'intervention médicale. La prise en compte du niveau de litteratie du patient peut aider le médecin à mieux comprendre les obstacles que rencontre ce dernier dans la prise en charge 
Tableau III. Recommandations pédagogiques pour atténuer les obstacles communicationnels liés au faible taux de littératie du patient.

\begin{tabular}{|l|l|}
\hline $\begin{array}{l}\text { Créer un environnement convivial et chaleu- } \\
\text { reux }^{[10]}\end{array}$ & $\begin{array}{l}\text { - Adapter l'établissement au faible taux de litté- } \\
\text { ratie du patient (flèches au sol pour guider le pa- } \\
\text { tient). } \\
\text { - Faire preuve de patience. }\end{array}$ \\
\hline Être à l'affût des signes de faible littératie $^{[7]}$ & $\begin{array}{l}\text { - Certains signes ne trompent pas : difficulté du pa- } \\
\text { tient à remplir des formulaires, amène une tierce } \\
\text { personne pour se faire expliquer les consignes, } \\
\text { évite de lire ou semble anxieux en lisant, etc. }\end{array}$ \\
\hline $\begin{array}{l}\text { Fournir des informations écrites ou orales adap- } \\
\text { tées au faible taux de littératie du patient }{ }^{[10]}\end{array}$ & $\begin{array}{l}\text { - Vulgariser l'information en utilisant des mots } \\
\text { courants exempts de jargon, en faisant des phrases } \\
\text { simples. }\end{array}$ \\
& $\begin{array}{l}\text { - S'adresser directement à l'interlocuteur en privi- } \\
\text { légiant la forme affirmative. }\end{array}$ \\
& $\begin{array}{l}\text { - Organiser l'information, isoler les éléments clefs } \\
\text { du message. }\end{array}$ \\
\hline Recourir au non-verbal ${ }^{[10]}$ & $\begin{array}{l}\text { - Joindre le geste à la parole. } \\
\text { - Regarder le patient dans les yeux. } \\
\text { - Faire une démonstration lorsque nécessaire (ex. } \\
\text { montrer comment utiliser un inhalateur). }\end{array}$ \\
\hline $\begin{array}{l}\text { Vérifier la compréhension et l'application de } \\
\text { l'information }^{[10]}\end{array}$ & $\begin{array}{l}\text { - Faire répéter la personne pour vérifier sa com- } \\
\text { préhension. } \\
\text { - Répéter le même message au besoin. } \\
\text { - Laisser du temps pour les questions. }\end{array}$ \\
\hline
\end{tabular}

de sa maladie, notamment s'il est atteint de diabète $^{[27]}$. Certains auteurs ont souligné l'importance de prendre en compte aussi le niveau de littératie des aidants naturels ${ }^{[28]}$. Des pistes de solutions ont été proposées pour surmonter les obstacles dans le système de santé, liés au faible taux de littératie. La nécessité que la communication entre le médecin et son patient exploite des stratégies adaptées est un point crucial et mérite aujourd'hui d'être explicitement prise en compte. Le tableau III rapporte quelques recommandations pédagogiques qui peuvent guider le clinicien dans son interaction avec un patient ayant un niveau de littératie relativement bas.

Plusieurs auteurs ou experts en littératie préconisent, par exemple : l'utilisation d'un langage dépourvu du jargon médical, l'utilisation d'un minimum d'idées clefs dans la discussion, l'interrogation du patient sur ce qu'il a compris de son régime de soin, de sa prise de traitement, l'incitation à poser des questions et dans certains cas, la multiplication des modalités de communication (illustrations, communication écrite et orale) ${ }^{[1,10,16,19,29,30]}$. Il semble que la méthode de transmission de l'information la plus appréciée par les patients soit encore les consultations en face-à-face et que cette méthode soit très bénéfique dans le traitement des personnes malades ayant un faible taux de littératie ${ }^{[27,31]}$. En effet, les patients attachent d'autant plus importance à la communication personnelle avec leur médecin qu'ils ont un faible taux de littératie ${ }^{[31]}$.

La dimension culturelle de la littératie en santé et son lien avec la relation patient-médecin sont également bien documentés ${ }^{[26,32]}$. Des recommandations pour surmonter certaines barrières communicationnelles ont été formulées : parfaire les compétences culturelles, adapter la communication aux différences culturelles ${ }^{[1,26]}$ et fournir des interprètes ou des médiateurs culturels ${ }^{[1,33]}$. Le médecin compétent culturellement doit être sensible et 
vigilant aux différences ethniques, notamment en ce qui concerne les tests de dépistage, les prescriptions, les procédures et la fourniture des résultats de santé ${ }^{[26]}$. Ainsi, la formation à la communication avec le patient devrait être intégrée systématiquement aux différentes formations que reçoit le médecin et elle ne devrait pas constituer un élément d'apprentissage à part. En somme, pour chacune des formations dispensées, le formateur devrait poser la question suivante aux participants : «Et maintenant, comment allez-vous en parler à votre patient?»

\section{Contributions}

Contributions : Claude Richard et Marie-Thérèse Lussier ont participé conjointement et solidairement à la rédaction du manuscrit.

\section{Références}

1. Rootman I, Gordon-El-Bihbety D. Vision d'une culture de la santé au Canada : Rapport du groupe d'experts sur la littératie en matière de santé. Ottawa : Association canadienne de santé publique, 2008.

2. Gauthier J. Les patients aux prises avec des problèmes d'alphabétisme fonctionnel. In : Richard C, Lussier MT (Éds). La communication professionnelle en santé. Montréal : ERPI, 2005:401-426.

3. Tuijnman A. Évaluation de la littératie des adultes en Amérique du Nord : Étude comparative internationale. Ottawa : Statistique Canada, 2001.

4. Kirsch IS, Jungeblut A, Jenkins L, Kolstad A. Adult literacy in America: A First Look at the results of the National Adult Literacy Survey (NALS). Washington (DC): National Center for Education Statistics, United States Departement of Education, 1993.

5. Statistique Canada et Ressources humaines et développement des compétences Canada. Miser sur nos compétences, résultats canadiens sur l'enquête internationale sur l'alphabétisation et les compétences des adultes. Ottawa : Ministère de l'industrie, 2005.

6. National Literacy Secretariat (NLS), \& Secrétariat national de l'alphabétisation. Document de base sur l'Enquête internationale sur l'alphabétisation des adultes, 2008 [Online] Disponible sur : http:// www.bdaa.ca/biblio/recherche/sna/ialsf/ ialsrepf/ialsfrp2/cover.htm
7. Conseil canadien sur l'apprentissage. État de l'apprentissage au Canada : Pas le temps de s'illusionner. Rapport sur l'apprentissage au Canada. Ottawa, Canada, 2007.

8. Institute of Medicine. Health literacy: A prescription to end confusion. Washington, DC 20001: National Academies Press, 2004.

9. OCDE et statistique Canada. La littératie à l'ère de l'information : Rapport final de l'enquête internationale sur la littératie des adultes (EILA), Paris, 2000.

10. Ouellette C, Médieros I, Thouin M. Alphabétisme, santé et médicaments : des liens importants à connaître et à comprendre. In Lévy JJ, Garnier C (Eds). La chaîne des médicaments. Perspectives pluridisciplinaires. Montréal (QC) : Presses de l'Université du Québec, 2007.

11. Agness C, Murrell E, Nkansah N, Martin CM. Poor health literacy as a barrier to patient care. Consult Pharm 2008;23:378-82.

12. Association canadienne de santé publique. De bons remèdes pour les aînés : lignes directrices sur la rédaction des documents et la conception des emballages des médicaments sur ordonnances. Ottawa : ACSP, 2002.

13. Davis TC, Williams MV, Marin E, Parker RM, Glass J. Health Literacy and Cancer Communication. CA Cancer J Clin 2002;52:134-49.

14. Schillinger D, Bindman A, Wang F, Stewart A, Piette J., Functional health literacy and the quality of physicianpatient communication among diabetes patients. Patient Educ Couns 2004;52:315-23.

15. Schillinger D, Grumbach K, Piette J, Wang F, Osmond, D, Daher C, Palacios J, Diaz Sullivan G, Bindman AB. Association of health litteracy with diabetes outcomes. JAMA 2002;288:475-82.

16. Villaire M, Mayer G. Low health literacy: the impact on chronic illness management. Prof Case Manag 2007;12:213-6.

17. Downey LV, Zun LS. Assessing adult health literacy in urban healthcare settings. J Natl Med Assoc 2008;100:1304-8.

18. Tang YH, Pang SMC, Chan MF, Yeung GSP, Yeug VTF. Health literacy, complication awareness, and diabetic control in patients with type 2 diabetes mellitus. J Adv Nurs 2008;62:74-83.

19. Hironaka LK, Paasche-Orlow MK. The implications of health literacy on patient-provider communication. Arch Dis Child 2008;93:428-32.

20. Williams VM, Davis T, Parker RM, Weiss BD. The role of health literacy in patient-physician communication. Fam Med 2002;34:383-9. 
21. Persell SD, Osborn CY, Richard R, Skripkauskas S, Wolf MS. Limited health literacy is a barrier to medication reconciliation in ambulatory care. J Gen Intern Med 2007;22:1523-6.

22. Schwartzberg JG, Cowett A, VanGeest J, Wolf MS. Communication techniques for patients with low health literacy: a survey of physicians, nurses, and pharmacists. Am J Health Behav 2007;31 suppl1:S96-104.

23. Castro CM, Wilson C, Wang F, Schillinger D. Babel babble: physicians' use of unclarified medical jargon with patients. Am J Health Behav 2007;31 Suppl 1:S85-95.

24. Young, M.E., G.R. Norman, Humphreys KR. The role of medical language in changing public perceptions of illness. PLoS ONE, 2008;3:e3875.

25. Baker DW, Wolf MS, Feinglass J, Thompson JA, Guzmararian JS, Huang P. Health literacy and mortality among ederly persons. Arch Intern Med 2007;167:1503-9.

26. Flores, G. Culture and the patient-physician relationship: Achieving cultural competency in health care. Journal Pediatr 2000;136:14-23.

27. Ishikawa, H, Takeuchi T, Yano E. Measuring functional, communicative, and critical health literacy among diabetic patients. Diabetes Care 2008; 31:874-9.

28. Bevan JL, Pecchioni LL.Understanding the impact of family caregiver cancer literacy on patient health outcomes. Patient Educ Couns 2008;71:356-64.
29. Logan RA. Clinical, classroom, or personal education: attitudes about health literacy. J Med Libr Assoc 2007;95:127-37.

30. Richard C, Lussier MT. La littératie en santé : Une chance sur deux d'être compris par son patient. Les cahiers de MedActuel 2008;8:35-6.

31. Shaw, A, Ibrahim S, Reid F, Ussher M, Rowlands G. Patients' perspectives of the doctor-patient relationship and information giving across a range of literacy levels. Patient Educ Couns 2009;75:114-20.

32. Zanchetta MS, Poureslami IM.Health literacy within the reality of immigrants' culture and language. Can J Public Health 2006;97 Suppl 2:S26-30.

33. Harmsen JA, Bernsen RM, Bruijnzeels MA, Meeuwesen L. Patients' evaluation of quality of care in general practice: what are the cultural and linguistic barriers? Patient Educ Couns 2008;72:155-62.

Correspondance et offprints : Claude Richard, Équipe de recherche en soins de première ligne, Cité de la santé de Laval Centre de santé et de services sociaux de Laval 1755, Bd RenéLaennec, H7M 3L9 Laval, Québec, Canada Mailto : ell4400@videotron.ca 